Mathématiques et sciences humaines
Mathematics and social sciences

138 | Été 1997

Varia

\title{
Dissimilarités multivoies et généralisations d'hypergraphes sans triangles
}

Multiway dissimilarities and generalizations of hypergraphs with no triangles

Jean Diatta

\section{(2) OpenEdition}

1 Journals

Édition électronique

URL : http://journals.openedition.org/msh/2753

DOI : $10.4000 / \mathrm{msh} .2753$

ISSN : 1950-6821

Éditeur

Centre d'analyse et de mathématique sociales de l'EHESS

Édition imprimée

Date de publication : 1 mars 1997

ISSN : 0987-6936

Référence électronique

Jean Diatta, "Dissimilarités multivoies et généralisations d'hypergraphes sans triangles »,

Mathématiques et sciences humaines [En ligne], 138 | Été 1997, mis en ligne le 10 février 2006, consulté le 23 juillet 2020. URL : http://journals.openedition.org/msh/2753 ; DOl : https://doi.org/10.4000/msh. 2753

Ce document a été généré automatiquement le 23 juillet 2020.

(c) École des hautes études en sciences sociales 


\section{Dissimilarités multivoies et généralisations d'hypergraphes sans triangles}

Multiway dissimilarities and generalizations of hypergraphs with no triangles

Jean Diatta

\section{RÉSUMÉS}

Les dissimilarités multivoies sont une généralisation naturelle des dissimilarités usuelles deux voies. Dans ce papier, des classes de dissimilarités multivoies sont étudiées, ainsi que des modèles de passage d'un nombre de voies donné à un autre nombre de voies. Une application à la spécification de systèmes classifiants a conduit à une bijection entre une classe de dissimilarités multivoies et une famille de systèmes stratifiés de classification.

Multiway dissimilarities naturally generalize the usual two-way dissimilarities. In this paper, particular classes of multiway dissimilarities are studied as well as some models for deriving from a dissimilarity of a given number of ways, one of a greater or a lower number of ways. An application to clustering systems specification has led to a one-to-one correspondence between a class of multiway dissimilarities and a family of stratified clustering systems.

INDEX

Thèmes : classification - partition, distances, graphes, ordres et préordres 